\title{
ON WEIGHTED ESTIMATES FOR STEIN'S MAXIMAL FUNCTION
}

\author{
Hendra Gunawan
}

Let $\phi$ denote the normalised surface measure on the unit sphere $S^{n-1}$. We shall be interested in the weighted $L^{p}$ estimate for Stein's maximal function $M_{\phi} f$, namely

$$
\left\|M_{\phi} f\right\|_{L^{p}(w)} \leqslant C_{p, w}\|f\|_{L P(w)}, \quad f \in L^{p}(w)
$$

where $w$ is an $A_{p}$ weight, especially for $1<p \leqslant 2$. Using the Mellin transformation approach, we prove that the estimate holds for every weight $w^{\delta}$ where $w \in A_{p}$ and $0 \leqslant \delta<(p(n-1)-n) /(n(p-1))$, for $n \geqslant 3$ and $n /(n-1)<p \leqslant 2$.

\section{INTRODUCTION}

Let $\phi_{r}$ be the normalised surface measure on the sphere of radius $r$, centre 0 in $\mathbf{R}^{n}$. Consider Stein's maximal function $M_{\phi} f$, which is defined by

$$
M_{\phi} f(x)=\sup _{r>0}\left|\phi_{r} * f(x)\right|, \quad x \in \mathbf{R}^{n}
$$

for any nice function $f$ on $\mathbf{R}^{n}$. Then we have the $L^{p}$ inequality

$$
\left\|M_{\phi} f\right\|_{p} \leqslant C_{p}\|f\|_{p}, \quad f \in L^{p}
$$

for $n \geqslant 2$ and $n /(n-1)<p \leqslant \infty$, which has been shown to be best possible $[1,4]$. In this paper, we are interested in the weighted $L^{p}$ estimate for Stein's maximal function,

$$
\left\|M_{\phi} f\right\|_{L^{p}(w)} \leqslant C_{p, w}\|f\|_{L^{p}(w)}, \quad f \in L^{p}(w)
$$

where $w \in A_{p}$, especially for $1<p \leqslant 2$. (Consult [3] about $A_{p}$ weights.) For $n \geqslant 3$, a positive result can be found in [3]; here we shall reprove and extend it.

\section{Received 28th August, 1995}

This work was initiated when the author visited the School of Mathematics, University of New South Wales, Sydney, Australia, in June 1994. Many thanks are due to Professor M. Cowling who suggested the problem and helped the author with a lot of ideas. Partial support from the Centre for Mathematics, ITB, Bandung, is also acknowledged.

Copyright Clearance Centre, Inc. Serial-fee code: 0004-9729/96 \$A2.00+0.00. 
Using the Mellin transformation approach of Cowling and Mauceri [2], let $K_{u}(x)=$ $C(u)|x|^{-n+i u}$, where $C(u)=\pi^{-(n / 2)+i u} \Gamma(n-i u / 2) / \Gamma(i u / 2) .\left(K_{u}\right.$ is the distribution on $\mathbf{R}^{n}$ whose Fourier transform is $\widehat{K_{u}}(\xi)=|\xi|^{-i u}$.) Then, formally, we have

$$
\phi(x)=P_{1}(x)+\int_{\mathbf{R}} D(u) K_{u}(x) d u, \quad x \in \mathbf{R}^{n}
$$

where $P_{1}$ denotes the Poisson kernel at 1 and $D(u)$ satisfies

$$
2 \pi C(u) D(u)=\int_{0}^{\infty}\left(\omega_{n-1}^{-1} \delta_{1}-P_{1}\right)(s) s^{n-1-i u} d s, \quad u \in \mathbf{R},
$$

with $\delta_{1}$ being the point mass at 1 . One may observe that $C(u)=O\left((1+|u|)^{n / 2}\right)$ and $D(u)=O\left((1+|u|)^{-(n / 2)}\right)$. Now, for every $r>0$,

$$
\phi_{r}(x)=P_{r}(x)+\int_{\mathbf{R}} D(u) K_{u}(x) r^{-i u} d u, \quad x \in \mathbf{R}^{n}
$$

and accordingly, for every smooth function $f$ on $\mathbf{R}^{n}$,

$$
\phi_{r} * f(x)=P_{r} * f(x)+\int_{\mathbf{R}} D(u) K_{u} * f(x) r^{-i u} d u, \quad x \in \mathbf{R}^{n} .
$$

Hence

$$
M_{\phi} f(x) \leqslant M_{P_{1}} f(x)+\int_{\mathbf{R}}|D(u)|\left|K_{u} * f(x)\right| d u, \quad x \in \mathbf{R}^{n} .
$$

Here $M_{P_{1}} f(x)=\sup _{r>0}\left|P_{r} * f(x)\right|$ for $x$ in $\mathbf{R}^{n}$. Since we know that $M_{P_{1}} f$ is majorised by the Hardy-Littlewood maximal function $M_{\mathrm{HI}} f$, we obtain

$$
\left\|M_{\phi} f\right\|_{L^{p}(w)} \leqslant\left\|M_{\mathrm{HL}} f\right\|_{L^{p}(w)}+\int_{\mathbf{R}}|D(u)|\left\|K_{u} * f\right\|_{L^{p}(w)} d u .
$$

Thus, to verify the estimate, we need to get a good weighted $L^{p}$ estimate for $K_{u} * f$, that is one that makes

$$
\int_{\mathbf{R}}|D(u)|\left\|K_{u} * f\right\|_{L^{p}(w)} d u \leqslant C_{p, w}\|f\|_{L^{p}(w)},
$$

for $1<p \leqslant 2$. 


\section{MaIN RESULTS}

We obtain the following results. The first lemma below is standard.

Lemma 1. For $|x| \geqslant 2|y|$ and for all $\gamma \in(0,1)$,

$$
\left|K_{u}(x-y)-K_{u}(x)\right| \leqslant C(1+|u|)^{(n / 2)+\gamma}|y|^{\gamma}|x|^{-n-\gamma} .
$$

PrOOF: For $|x| \geqslant 2|y|$, we have, as in [2], two estimates

$$
\left|K_{u}(x-y)-K_{u}(x)\right| \leqslant C(1+|u|)^{n / 2}|x|^{-n}
$$

and

$$
\left|K_{u}(x-y)-K_{u}(x)\right| \leqslant C(1+|u|)^{(n / 2)+1}|y||x|^{-n-1} .
$$

Interpolating these estimates, we get

$$
\left|K_{u}(x-y)-K_{u}(x)\right| \leqslant C(1+|u|)^{(n / 2)+\gamma}|y|^{\gamma}|x|^{-n-\gamma},
$$

for all $\gamma \in(0,1)$.

Following the work of Watson [6], we have

Lemima 2. For $1<p \leqslant 2$ and for any $\gamma \in(0,1)$,

$$
\left\|K_{u} * f\right\|_{L^{p}(w)} \leqslant C_{p, w, \gamma}(1+|u|)^{(n / 2)+\gamma}\|f\|_{L^{p}(w)}, \quad f \in L^{p}(w),
$$

whenever $w \in A_{p}$.

Proof: First note that $\left|\widehat{K}_{u}(\xi)\right|=1$ for all $\xi \in \mathbf{R}^{n}$. Next, we need to show that the $L^{r}$-Hörmander condition : for $R>2|y|>0$,

$$
\sum_{j=1}^{\infty}\left(2^{j} R\right)^{n / r^{\prime}}\left(\int_{2^{j} R<|x|<2^{j+1} R}\left|K_{u}(x-y)-K_{u}(x)\right|^{r} d x\right)^{1 / r} \leqslant C_{\gamma}(1+|u|)^{(n / 2)+\gamma},
$$

is satisfied for all $r \in(1, \infty)$. (Here $r^{\prime}$ denotes the dual exponent to $r$.) Having done this, we can then choose $r \in(1, \infty)$ sufficiently large such that $w^{r^{\prime}} \in A_{p}$. Thus, following [6], we obtain

$$
\left\|K_{u} * f\right\|_{L^{p}(w)} \leqslant C_{p, w, \gamma}(1+|u|)^{(n / 2)+\gamma}\|f\|_{L^{p}(w)}, \quad f \in L^{p}(w),
$$

as desired. Indeed, using Lemma 1 , we observe that for all $r \in(1, \infty)$,

$$
\begin{aligned}
& \int_{2^{j} R<|x|<2^{j+1} R}\left|K_{u}(x-y)-K_{u}(x)\right|^{r} d x \\
\leqslant & C^{r}(1+|u|)^{(n r / 2)+\gamma r}|y|^{\gamma r} \int_{2^{j} R<|x|<2^{j+1} R}|x|^{-n r-\gamma r} d x \\
\leqslant & C^{r}(1+|u|)^{(n r / 2)+\gamma r} R^{\gamma r} \int_{2^{j} R_{<t<2^{j+1} R}} t^{-n(r-1)-\gamma r}(d t) / t \\
\leqslant & C^{r}(1+|u|)^{(n r / 2)+\gamma r} R^{\gamma r}\left(2^{j} R\right)^{-n(r-1)-\gamma r} \\
= & {\left[C(1+|u|)^{(n / 2)+\gamma}\left(2^{j} R\right)^{-\left(n / r^{\prime}\right)_{2}} 2^{-\gamma j}\right]^{r} . }
\end{aligned}
$$


Therefore the condition is satisfied and the lemma is proved. (We have actually proved that the estimate holds whenever $w \in A_{p}$, for $1<p<\infty$.)

We are aware that the estimate in Lemma 2 is not good enough. We have, however, the following result of Cowling and Mauceri [2] for the unweighted case.

Lemma 3. (Cowling and Mauceri.) For $1<p \leqslant 2$ and for any $\gamma \in(0,1)$,

$$
\left\|K_{u} * f\right\|_{p} \leqslant C_{p, \gamma}(1+|u|)^{(n / p)-(n / 2)+\gamma}\|f\|_{p}, \quad f \in L^{p} .
$$

Now we have a better estimate for $K_{u} * f$, namely

Theorem 4. For $1<p \leqslant 2$ and for any $\gamma \in(0,1)$,

$$
\left\|K_{u} * f\right\|_{L^{p}\left(w^{\delta}\right)} \leqslant C_{p, w, \gamma, \delta}(1+|u|)^{(n / p)-(n / 2)+\delta n-(\delta n / p)+\gamma}\|f\|_{L^{p}\left(w^{\delta}\right)}, \quad f \in L^{p}\left(w^{\delta}\right),
$$

whenever $w \in A_{p}$ and $0 \leqslant \delta \leqslant 1$.

Proof: The proof follows directly from Lemma 2 and Lemma 3 by the Stein-Weiss interpolation theorem [5].

Theorem 4 leads us to the weighted $L^{p}$ estimate for Stein's maximal function.

THEOREM 5. For $n \geqslant 3$ and $n /(n-1)<p \leqslant 2$, the weighted $L^{p}$ estimate

$$
\left\|M_{\phi} f\right\|_{L^{p}\left(w^{\delta}\right)} \leqslant C_{p, w, \delta}\|f\|_{L^{p}\left(w^{\delta}\right)}, \quad f \in L^{p}\left(w^{\delta}\right),
$$

holds whenever $w \in A_{p}$ and $0 \leqslant \delta<(p(n-1)-n) /(n(p-1))$.

PROOF: Choose $\gamma \in(0,1)$ sufficiently small such that

$$
0 \leqslant \delta<(p(n-1-\gamma)-n) /(n(p-1)) .
$$

Then, by Theorem 4, we have

$$
\begin{aligned}
\int_{\mathbf{R}}|D(u)|\left\|K_{u} * f\right\|_{L^{p}\left(w^{\delta}\right)} d u & \leqslant C_{p, w, \delta}\|f\|_{L^{p}\left(w^{\delta}\right)} \int_{\mathbf{R}}(1+|u|)^{(n / p)-n+\delta n-(\delta n / p)+\gamma} d u \\
& \leqslant C_{p, w, \delta}\|f\|_{L^{p}\left(w^{\delta}\right)}
\end{aligned}
$$

and so the theorem follows immediately.

For power weights $w(x)=|x|^{a}$, we know that $w \in A_{p}$ for some $p>1$ if and only if $-n<a<n(p-1)$. So, Theorem 5 implies that the estimate holds for $w(x)=|x|^{a}$ with $-(p(n-1)-n) /(p-1)<a<p(n-1)-n$. Stating it in another way, the estimate with respect to $w(x)=|x|^{a}$ holds for $(n+a) /(n-1)<p \leqslant 2$ when $a \geqslant 0$, or for $(n+a) /(n+a-1)<p \leqslant 2$ when $a<0$. Thus, for $p \leqslant 2$, our result agrees with the one stated in $[3, p .571]$ for the special case where $w(x)=|x|^{a}$ with $a \geqslant 0$. 


\section{CONCLUding ReMarks}

We suspect that the same estimate also holds for $p>2$, but we encounter difficulties in verifying it. Duality arguments will not work since the endpoints of the range of allowable $p$ 's are not symmetric. The Stein-Weiss interpolation theorem only gives the estimate for $2 \leqslant p \leqslant \infty$ provided that $w \in A_{2}$ and $0 \leqslant \delta<(n-2) / n$. Also, since the estimate holds only for some but not all $w \in A_{p}$ when $n /(n-1)<p \leqslant 2$, we cannot use the existing extrapolation theorem of Rubio de Francia and Garcia-Cuerva. Some novel technique seems to be needed here and we are still working on it.

\section{REFERENCES}

[1] J. Bourgain, 'Averages in the plane over convex curves and maximal operators', J. Analyse Math. 47 (1986), 69-85.

[2] M. Cowling and G. Mauceri, 'On maximal functions', Rend. Sem. Mat. Fis. Milano 49 (1979), 79-87.

[3] J. Garcia-Cuerva and J.L. Rubio de Francia, Weighted norm inequalities and related topics (North-Holland, Amsterdam, 1985).

[4] E.M. Stein, 'Maximal functions: spherical means', Proc. Nat. Acad. Sci. U.S.A. 73 (1976), 2174-2175.

[5] E.M. Stein and G. Weiss, 'Interpolation of operators with change of measures', Trans. Amer. Math. Soc. 87 (1958), 159-172.

[6] D.K. Watson, 'Weighted estimates for singular integrals via Fourier transform estimates', Duke Math. J. 60 (1990), 389-399. 